Foaming Behaviors and Mechanical Properties of Injection Molded Polylactide/

\title{
Cotton-fiber Composites
}

Xiaoli Zhang ${ }^{1,2^{*}}$, Weidan Ding ${ }^{2}$, Eunse Chang ${ }^{2}$, Xuefeng Chen ${ }^{3}$, Jingbo Chen $^{1 *}$,

$$
\text { Chul B. Park }{ }^{2 *} \text {, Changyu Shen }{ }^{1}
$$

1 School of Materials Science and Engineering, Zhengzhou University, Zhengzhou, China 450001

2 Microcellular Plastics Manufacturing Laboratory, Department of Mechanical and Industrial Engineering,

University of Toronto, Toronto, Ontario, Canada M5S $3 G 8$

3 China National Pulp and Paper Research Institute, Beijing, China 100102

*Corresponding authors: zhangxl@zzu.edu.cn, chenjb@zzu.edu.cn

park@mie.utoronto.ca, Tel: +1 416-978-3053

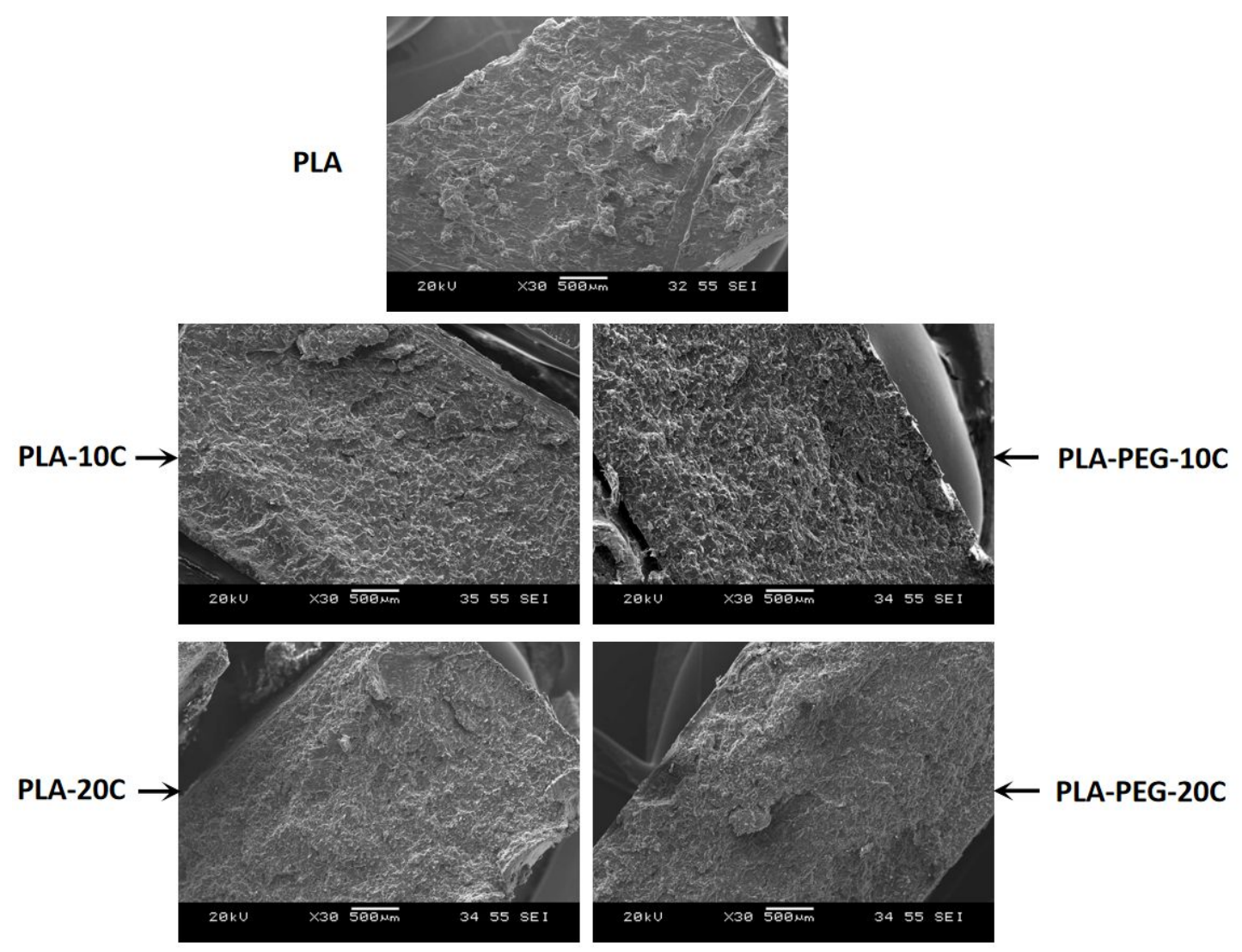

Figure S1 SEM pictures of solid neat PLA and PLA/Cotton- fibers at a full cavity filled level, and sampled at the middle of the plate (Point B in Figure 2) 

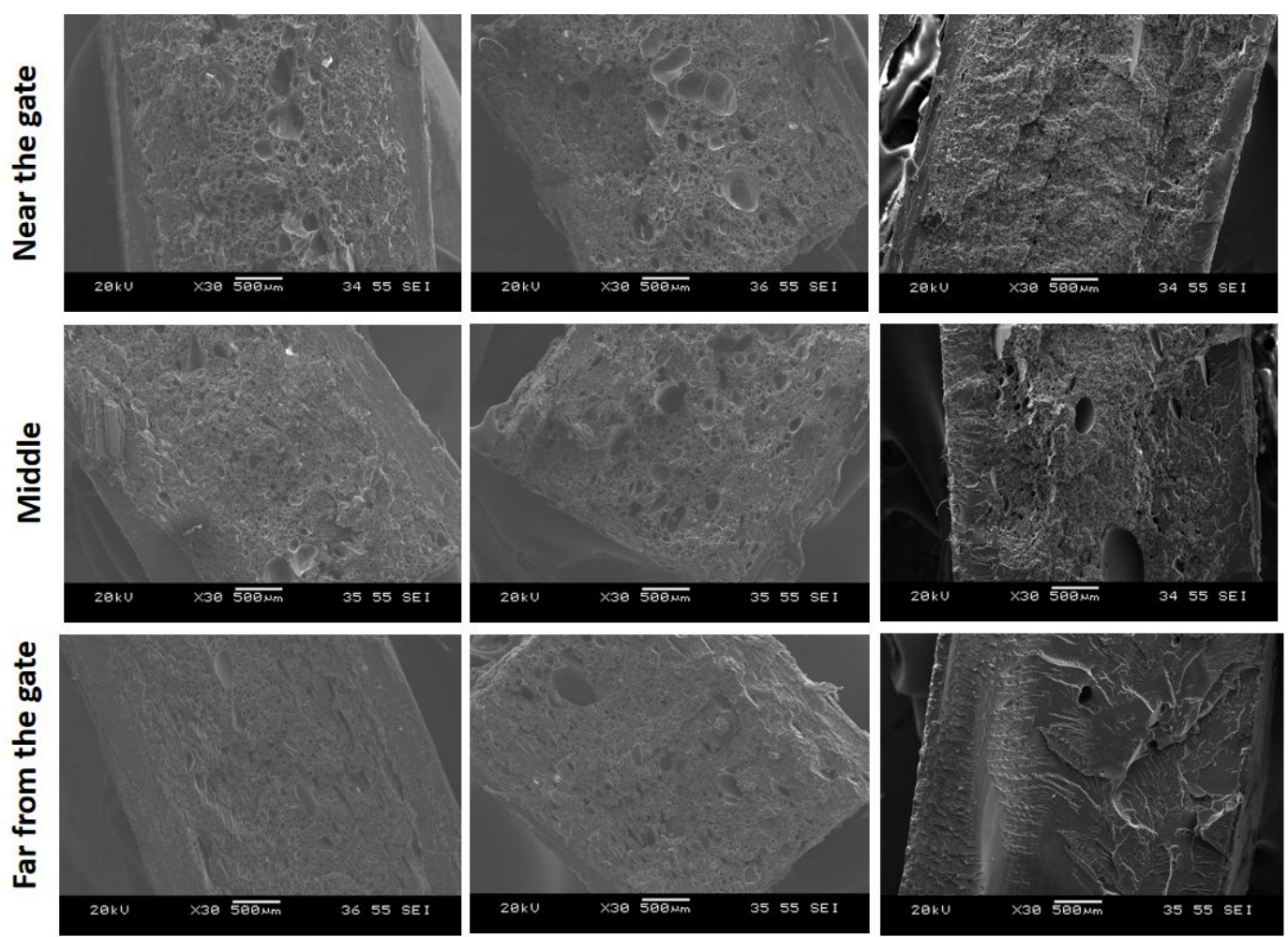

$70 \%$ filled

$80 \%$ filled

$100 \%$ filled

Figure S2 SEM pictures of foamed neat PLA at different cavity filled level
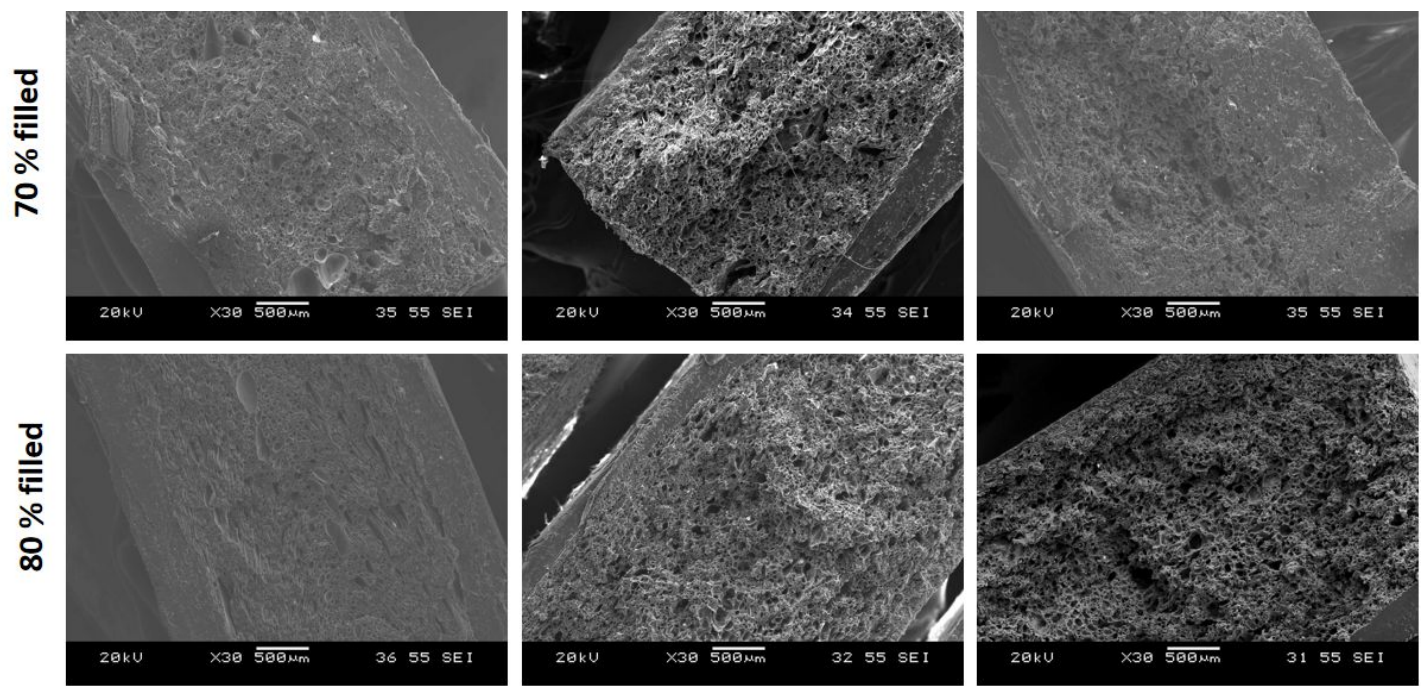

PLA-P

PLA-10C

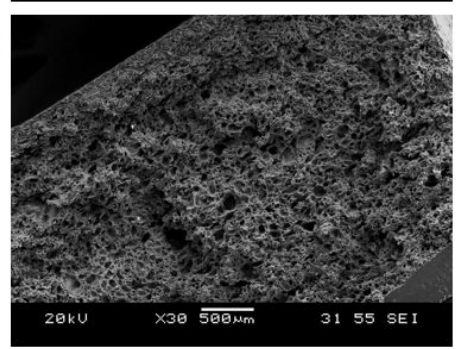

PLA-20C

Figure S3 SEM pictures of foamed PLA and PLA/Cotton- fibers at different cavity filled levels,

sampled at the middle of the plate (Point B in Figure 2) 